\title{
Termalismo e crenoterapia: potencialidades e desafios para a saúde coletiva no Brasil
}

Thermalism and Crenotherapy: potentialities and challenges for public health in Brazil Termalismo y Crenoterapia: potencialidades y desafíos para la salud colectiva en Brasil

\author{
Fernando Hellmann ${ }^{1}$; Lívia Crespo Drago ${ }^{2}$
}

\section{Resumo}

Dado o imenso potencial brasileiro, o termalismo social e a crenoterapia, previstas na Política Nacional de Práticas Integrativas e Complementares, são práticas pouco exploradas como fator de recuperação, manutenção e promoção da saúde. Reflete-se, neste artigo, as potencialidades e desafios do termalismo social e crenoterapia para a saúde coletiva no Brasil. Para tanto, aproximase aos conceitos de tais práticas, apresenta-se breve histórico do termalismo/crenoterapia no Brasil e no Sistema Único de Saúde, para então abordar alguns dos desafios existentes no campo. Considera-se que há pouco conhecimento dessa temática por parte das equipes de saúde da família e núcleos de apoio, os quais podem integrar algumas práticas termais/crenoterápicas na atenção básica, para além do termalismo clássico aplicado em termas. Um conjunto de ações, notadamente a formação em termalismo, pesquisa e financiamento, são discutidos com vistas ao fortalecimento destas práticas enquanto agente de promoção da saúde.
\end{abstract}

Descritores: Balneologia; Saúde Pública; Sistema Único de Saúde.

\begin{abstract}
Given the immense Brazilian potential, social thermalism and crenotherapy, included in the National Policy of Integrative and Complementary Practices, are little explored practices as a factor for recovery, maintenance and health promotion. The article discusses the potentialities and challenges of social thermalism/balneotherapy and crenotherapy for public health in Brazil. Therefore, we show concepts of such practices, a brief history of the thermalism/crenoterapia in Brazil and the Unified Health System, to address some of the challenges in the field. It is considered that there is little knowledge of this theme by family health teams and support groups, which can integrate some thermal/crenotherapeutic practices in basic care, in addition to the classic termalism applied in spas. A set of actions, notably training in balneotherapy, research and financing, are discussed with a view to strengthening these practices as a health promotion agent.
\end{abstract}

Key-words: Balneology; Public Health; Unified Health System.

\begin{abstract}
Resumen
Dado el inmenso potencial brasileño, el termalismo social y la crenoterapia, previstas en la Política Nacional de Prácticas Integrativas y Complementarias, son prácticas poco exploradas como factor de recuperación, mantenimiento y promoción de la salud. Se refleja, en este artículo, las potencialidades y desafíos del termalismo social y la crenoterapia para la salud colectiva en Brasil. Para ello, se aproxima a los conceptos de tales prácticas, se presenta brevemente histórico del termalismo/crenoterapia en Brasil y en el Sistema Único de Salud, para entonces abordar algunos de
\end{abstract}

\footnotetext{
${ }^{1} \mathrm{PhD}$. Universidade Federal de Santa Catarina (UFSC), Departamento de Saúde Pública. Campus Reitor João David Ferreira Lima, s/n, Trindade, Florianópolis - SC, Brasil. CEP: 88040-900. E-mail: hellmann.fernando@gmail.com

${ }^{2}$ MsC. Universidade do Sul de Santa Catarina (UNISUL), Curso de Natuologia.
} 
ISSN 2179-6750

los desafíos existentes en el campo. Se considera que hay poco conocimiento de esta temática por parte de los equipos de salud de la familia y núcleos de apoyo, los cuales pueden integrar algunas prácticas termales/crenoterápicas en la atención básica, además del termalismo clásico aplicado en termas. Un conjunto de acciones, especialmente la formación en termalismo, investigación y financiamiento, se discuten con vistas al fortalecimiento de estas prácticas como agente de promoción de la salud.

Palabras-claves: Balneología; Salud Pública; Sistema Único de Salud.

\section{Introdução}

Dada a abundância de águas minerais no Brasil, o termalismo e a crenoterapia são práticas de valor terapêutico pouco exploradas no país na atualidade. Ainda que se possa retratar longo histórico sobre o Termalismo no Brasil, o uso das águas mineiras e termais para o tratamento em saúde atrelado à saúde pública brasileira, como um direito do cidadão, é recente e insipiente. Mesmo com a institucionalização da Política Nacional de Práticas Integrativas e Complementares (PNPIC), o Termalismo Social/Crenoterapia é praticamente desconhecido pelas equipes atuantes na atenção básica, até mesmo nas cidades que possuem água termal natural. Passados dez anos da publicação da PNPIC ${ }^{1}$, a única diretriz desta política quanto ao Termalismo, ou seja, o incentivo à criação de Observatórios de Saúde onde atualmente são desenvolvidas experiências em Termalismo Social no âmbito do Sistema Único de Saúde (SUS), ficou bastante modesta.

Em comemoração aos dez anos da PNPIC ${ }^{1}$, este artigo pretende refletir acerca das potencialidades e desafios do Termalismo Social e crenoterapia para a saúde coletiva no Brasil. Para tanto, iniciamos com uma apresentação breve sobre as terminologias do uso da água em terapia, a qual ampliam as potencialidades do termalismo/crenoterapia na atenção básica; segue-se com breve relato histórico do termalismo/crenoterapia no Brasil; na sequência aborda-se a institucionalização do termalismo social/crenoterapia no SUS; por fim, alguns desafios são apresentados de modo a pensar a ampliação do Termalismo/Crenoterapia no SUS.

\section{Conceitos em termalismo social e crenoterapia}

O uso terapêutico da água para a saúde pode receber diversos nomes que variam de acordo com o tipo de água, a temperatura utilizada, a composição química, o modo de utilização e, às vezes, da categoria profissional que utiliza a água, como por exemplo: balneoterapia, crenoterapia, talassoterapia, crioterapia, hidroterapia, hidroginástica, termalismo, hidrologia médica ${ }^{2}$.

De maneira geral, a Balneoterapia é o descritor internacional em ciências da saúde para designar a terapia que utiliza banhos, geralmente quentes, em balneários de águas minerais 310 
ISSN 2179-6750

naturais para recuperação da saúde. Na França, o termo "Balneologia” é usado para se referir ao uso não medicinal de águas minerais, sendo crenoterapia o termo aplicado para o uso médico, preferindo-se o uso do termo Crenobalneoterapia ${ }^{3}$. De modo geral, alguns países latinos, tais como a Itália e Portugal, utilizam-se do termo "termalismo" como sinônimo de balneoterapia. Na Alemanha, bem como em outros países da Europa Central, Balneoterapia e ciência balneologia designam não apenas o uso de água mineral, independentemente da modalidade de uso (banho, ingestão, inalação e, em alguns casos, lavagens internas), mas também os pelóides (argilas) e gases naturais ${ }^{3}$. A partir desta breve aproximação aos termos é possível destacar que os brasileiros ligado às práticas integrativas, em especial no SUS, referem-se ao termalismo apenas aos tratamentos com águas, esquecendo-se dos pelóides, lamas, gases e outros recursos associados, tal como a educação terapêutica comumente feitos nas termas francesas, as quais integram o Termalismo/Balneologia.

Quanto ao uso da água em terapia, têm-se a talassoterapia, a qual se utiliza de recursos marinhos como a água do mar, algas e sais para tratamento. Crioterapia é a utilização da água em baixas temperaturas, comumente utilizada em fisioterapia. Hidroterapia pode ser tanto a aplicação de água em qualquer temperatura através de técnicas utilizadas por naturopatas clássicos e naturólogos, tais como compressas, cataplasmas, pacho, jatos de águas direcionados entre outras, como também a realização de exercícios orientados por fisioterapeutas dentro da água ${ }^{2}$. No Reino Unido, onde pouco se usa as águas minerais naturais como elemento terapêutico, o termo "Hidroterapia" é usado para descrever exercícios debaixo d'água enquanto que na Alemanha esse termo se aplica para tratamentos à base de água fria ou contrates, segundo o conceito de Kneipp (a mesma lógica da naturopatia clássica) ${ }^{3}$. Por sua vez, hidroginástica é a atividade física realizada dentro de meio aquoso sob supervisão do profissional de educação física ${ }^{2}$. Com o intuito de contornar os obstáculos quanto às nomenclaturas em termos de terapias pelas águas, publicou-se recentemente uma proposta para uma terminologia global ${ }^{4}$.

Já os nomes contemplados na PNPIC, Crenoterapia indica mais especificamente, a terapêutica do uso de água mineral natural em saúde e Termalismo a utilização de águas naturalmente termominerais para a promoção, prevenção, recuperação e reabilitação da saúde. São, portanto, termos praticamente sinônimos. Entretanto, a PNPIC qualifica como Termalismo "Social”. Esse termo já é utilizado desde a década de 1950 para expressar o sistema pelo qual o tratamento termal bastante, acessível à população abastada e pouco aos pobres, passou s ser ofertada de forma satisfatória e garantida a toda a população ${ }^{2,5}$. Termalismo Social é associado a incorporação pela seguridade de saúde, especialmente no período pós II Guerra Mundial, em que 
ISSN 2179-6750

viu-se nascer nos países europeus um modelo de Estado de bem-estar social que incorporava o tratamento termal nos sistemas de saúde. Ainda, a fim de ampliar o conceito de termalismo e abranger não apenas os aspectos de ampliação do acesso ao tratamento termal, como ainda os componentes da sustentabilidade do recuso natural e do desenvolvimento local, turismo e cultura, entende-se que o Termalismo Social ganha nova roupagem na saúde coletiva brasileira. Assim sendo, a promoção da saúde em termos do Termalismo Social ultrapassa os aspectos técnico da aplicação terapêutica da água e seus benefícios orgânicos para envolver a saúde em sua determinação social, demandando assim abordagem intersetorial e interdisciplinar, com vistas ao acesso à saúde como direito de cidadania ${ }^{2}$.

Faz-se necessário apontar a diferença entre a terapia de imersão em água potável propositalmente aquecida, portanto não necessariamente água mineral natural, do termalismo. A primeira, geralmente denominada hidroterapia, enfoca as qualidades físicas da água (empuxo, flutuação, densidade etc.), já a segunda enfoca as propriedades físicas, mas amplia e engloba também as propriedades químicas da água e suas finalidades terapêuticas ${ }^{2}$.

É importante frisar que o Termalismo/balneoterapia pode ser feito através do aquecimento das águas minerais cuja temperatura na fonte são frias. Ou seja, não apenas onde existem águas naturalmente quentes é que se pode utilizar o termalismo/crenoterapia na atenção básica. Havendo características físico-químicas em níveis que conferem atividade terapêutica, o aquecimento das águas pode ser realizado de modo a ampliar os efeitos terapêuticos, como por exemplo por intermédio de painéis solares, segundo as necessidades de práticas de cuidados específicos. Certamente, em locais com águas termominerais naturais a relação custo-benefício é maior do que aquecer a água, especialmente em se tratando de um balneário clássico. Contudo, algumas práticas podem ser feitas com pouca água, como o caso das nebulizações com águas minerais específicas as quais são fáceis de serem aplicadas na atenção básica, ainda que não se tenha uma terma no município. Este entendimento, ainda pouco explorado na crenoterapia brasileira, faz com que as potencialidades do uso das águas minero-medicinais sejam ampliadas para mais localidades.

\section{Breve histórico do termalismo e crenoterapia no Brasil}

A existência de águas termais foi relatada por colonizadores que exploravam o Brasil Colônia. No país, a primeira fonte de água termal foi encontrada pelos portugueses no ano de 1722, descrita por Bartolomeu Bueno da Silva Filho, quando da exploração de ouro e prata em Goiás ${ }^{6}$. Importantes fontes hidrominerais brasileiras foram descobertas ainda no século XVIII $6-8$. 
ISSN 2179-6750

Entretanto, a institucionalização do uso das águas termais no Brasil veio somente em 1818, com o decreto de Dom João VI, Rei do Reino Unido de Portugal, Brasil e Algarves especificadamente para a fonte de Caldas da Imperatriz (Santa Catarina), onde ali deveria ser construído um hospital termal aos moldes do hospital de Caldas da Rainha (Portugal), datado de 1485, uma vez que no país ainda não havia legislação sobre o termalismo. Este decreto é considerado o marco do termalismo no Brasil, na perspectiva de prática terapêutica ${ }^{7}$. Em 1848, D. Pedro II, Imperador do Brasil, instituiu a estância hidromineral de Caldas do Cubatão, posteriormente rebatizado como Caldas da Imperatriz, marcando o início da utilização de águas minerais em balneários ${ }^{9}$.

Em 1877 é instalado na cidade de Petrópolis o Imperial Estabelecimento Hidroterápico para realizar tratamentos, especialmente, à família imperial, tendo Dom Pedro II como frequentador assíduo ${ }^{10}$. Nota-se que a família real teve forte influência na propagação no Brasil da cultura europeia acerca do uso das águas termais.

A intensificação do surgimento das estâncias hidrominerais no Brasil se deu nas primeiras décadas do século XX, quando o Brasil já era uma república, e ordenava os planos urbanísticos das cidades criadas a partir da exploração das fontes ternais. Várias cidades nos estados de Goiás, Minas Gerais, Rio de Janeiro e São Paulo foram criadas nessa época. As estações termais eram glamorosas e apresentavam referências arquitetônicas das estações termais europeias como Vichy, na França; Baden-Baden, na Alemanha; Aix-les-Bains, Bagnères-de-Luchon, na França; e Montecatini, na Itália e eram frequentadas pela aristocracia da época, especialmente, por políticos, fazendeiros e comerciantes ${ }^{10,11}$.

Acompanhando esse desenvolvimento, as escolas de medicina da Universidade Federal de Minas Gerais (UFMG) e da Universidade Federal do Rio de Janeiro (UFRJ) incluíram em seus currículos o conteúdo de hidrologia médica: na UFMG, havia uma disciplina específica chamada de hidrologia e climatologia e na UFRJ, o conteúdo era desenvolvido na disciplina de terapêutica geral ${ }^{7}$.

O apogeu das estancias hidrominerais aconteceu entre os anos 1930 e 1940 por conta de seus aspectos terapêuticos e lúdicos, muito associado aos cassinos que haviam em anexo às estancias ${ }^{7}$. Em 1945, a fim de realizar a gestão da água, o Ministério de Minas e Energia, através do Departamento Nacional de Produção Mineral (DNPM), instituiu o Decreto-Lei ${ }^{\circ}$ 7.841, de 8 de agosto de 1945 que classifica as águas minerais brasileiras, regulamentando sua pesquisa, exploração, industrialização e comercialização, válido ainda nos dias atuais.

Entretanto, ao longo dos anos, busca pelo entretenimento nas estâncias acabou sendo 313 
ISSN 2179-6750

mais procurado do que os próprios tratamentos, o que mais tarde implicou em uma das razões do declínio desta atividade dada a proibição de jogos no país, em $1946^{7}$. A partir da década seguinte, 1950, houveram outros dois eventos que também contribuíram para o declínio: a intensificação dos investimentos imobiliários na orla marítima, deixando as águas termais em detrimento da água do mar; e o desenvolvimento da indústria farmacêutica que, com os corticoides e os antibióticos, induziu a substituição dos recursos naturais (neste caso a água) pelo uso de drogas com efeitos mais rápidos e potentes ${ }^{12}$. Assim, paulatinamente, as águas termais, como recurso terapêutico, foram sendo preteridas.

Atualmente, o turismo de lazer são as principais atividades das estâncias hidrominerais no Brasil. Entretanto, com a instituição da Política Nacional de Práticas Integrativas e Complementares em 2006, que estabelece, entre outras práticas, o termalismo social e crenoterapia ${ }^{1}$, há um panorama favorável para resgatar o uso das águas termais para a saúde e ampliar o acesso dessa prática aos usuários do SUS.

\section{Termalismo e Crenoterapia no Sistema Único de Saúde}

A VIII Conferência Nacional de Saúde foi indiscutivelmente um marco da Reforma Sanitária Brasileira em 1986, pois além de ter apresentado a base do que foi posteriormente instituído como Sistema Único de Saúde (SUS) na Constituição Brasileira em 1988, e também documentou a necessidade de introdução de Práticas Integrativas e Complementares (PIC) na assistência à saúde para que o usuário do sistema pudesse ter maior autonomia de escolha terapêutica.

Pensando em oportunizar esta escolha terapêutica pelo usuário do SUS a Comissão Interministerial de Planejamento e Ordenação (CIPLAN), constituída pelos ministérios da saúde, previdência e assistência social, educação e trabalho, criou em 1988 as normas e as diretrizes para a implementação da homeopatia, acupuntura, técnicas alternativas em saúde mental, termalismo e fitoterapia ${ }^{13}$.

Especificamente, em relação ao uso da água como recurso terapêutico, o Conselho Nacional de Saúde elaborou a resolução $\mathrm{n}^{\circ}$ 343, de 07 de outubro de 2004, para definir as ações governamentais que incluem a revalorização dos mananciais das águas minerais para o uso terapêutico, o delineamento de mecanismos de prevenção, fiscalização, controle e o incentivo à realização de pesquisas acerca da temática. Entretanto, foi somente em 2006 que o Ministério da Saúde, para atender as orientações da Organização Mundial da Saúde ${ }^{14}$ instituiu a Política Nacional de Práticas Integrativas e Complementares (PNPIC), cujo um dos objetivos é incorporar e 
ISSN 2179-6750

implementar a PNPIC no SUS, na perspectiva da prevenção de agravos e da promoção e recuperação da saúde, com ênfase na atenção básica, voltada para o cuidado continuado, humanizado e integral em saúde. As práticas inicialmente preconizadas pelo foram: Medicina Tradicional Chinesa-Acupuntura, Homeopatia, Fitoterapia/Plantas Medicinais, Medicina Antroposófica e o Termalismo Social/Crenoterapia ${ }^{13}$.

Especificadamente, sobre o Termalismo Social/Crenoterapia a PNPIC teve como única diretriz o incentivo à criação de Observatórios de Saúde onde atualmente são desenvolvidas experiências em Termalismo Social, no âmbito do SUS ${ }^{1}$. Dessa forma, pretende-se reunir as ações/atividades incipientes de termalismo no sistema de saúde para melhor compreender como de fato implementar essa prática.

Apesar de o Tratamento Termal/Crenoterápico ainda ser incipiente, o Ministério da Saúde, em 11 de janeiro de 2017 a partir da portaria $n^{\circ}$ 145, altera os procedimentos na Tabela de Procedimentos, Medicamentos, Órteses, Próteses e Materiais Especiais do SUS para atendimento na Atenção Básica. Desse modo, o Tratamento Termal/Crenoterápico está incluso na Forma de Organização 05 - Práticas Integrativas/Complementares do Grupo 03 - Procedimentos Clínicos, Subgrupo 09 - Terapias Especializadas com o sob código 03.09.05.006-5. Esta medida ajudará a dar ainda mais visibilidade aos o Tratamento Termal/Crenoterápico realizados na rede de atenção à saúde.

Convém destacar que o Edital de Chamamento Público no 5 para o Fortalecimento das Ações de Serviços de Práticas Integrativas e Complementares no SUS, publicado em 2013 pela Coordenação de Áreas Técnicas do Departamento de Atenção Básica da Secretaria de Atenção à Saúde do Ministério da Saúde, contemplou o projeto "Termalismo Social na Atenção Básica Catarinense: realidade, sensibilização e ação” da Secretaria de Estado da Saúde de Santa Catarina, em parceria com o Curso de Naturologia da Universidade do Sul de Santa Catarina e o Departamento de Saúde Pública da Universidade Federal de Santa Catarina. O projeto, o qual recebeu recursos aprovados pelo Ministério da Saúde, convênio n ${ }^{\circ} 795180 / 2013$, publicado no Diário Oficial da União em 09/01/2014, realizou diagnostico situacional das ações de termalismo desenvolvidas na atenção básica, sensibilização de profissionais e gestores de municípios com águas termominerais, bem como a publicação de material informativo e livro sobre o tema. A partir deste projeto, ações de qualificação e divulgação das potencialidades em termalismo começaram a ser implementados no Estado, bem como as ações/atividades incipientes de termalismo no sistema de saúde estão sendo incentivadas com intuito de serem acompanhadas para melhor compreender como de fato implementar essa prática na Atenção Básica e, quem sabe, em nível secundário. 
ISSN 2179-6750

Experiências em termalismo social e crenoterapia tem sido desenvolvida na atenção básica em algumas cidades brasileiras ${ }^{15}$, embora são poucas as realizadas que estão descritas na Comunidade de Práticas do Ministério da Saúde. De modo geral, a incipiência de experiências em termalismos na atenção básica é em parte condicionada pelas dificuldades que precisam ser enfrentadas, notadamente a formação qualificada de profissionais, bem como modelos de sustentabilidade de tais iniciativas.

\section{Desafios do Termalismo e Crenoterapia no Brasil}

O termalismo social/crenoterapia como práticas de promoção da saúde pode contribuir com a resolubilidade do SUS e ampliação do acesso equitativo às práticas termais em saúde com qualidade, eficácia, eficiência e uso multiprofissional seguro, bem como promover a saúde e o desenvolvimento sustentável ${ }^{2}$. O potencial do Termalismo e da Crenoterapia no Brasil pode ser percebido com a dimensão dos recursos hídricos, notadamente as águas minerais e termais. No entanto, desafios do Termalismo Social e da Crenoterapia são visíveis para a atenção básica, especializada, e até mesmo em sentido de desenvolvimento sustentável dos municípios turísticos com estâncias termais. Dentre os desafios, destacam-se: formação profissional qualificada; ampliação das pesquisas sobre o Termalismo e Crenoterapia; atualização e aprimoramento da legislações e fortalecimento de políticas públicas para a área; fortalecimento dos estabelecimentos de saúde com termalismo e crenoterapia na saúde pública; o desafio do financiamento.

A publicação da PNPIC em $2006^{1}$, o Termalismo e a Crenoterapia passam a integrar uma política nacional de saúde e com ela o aumento do interesse público sobre a temática. Porém, talvez o maior desafio brasileiro do termalismo social/crenoterapia se deriva da baixa qualificação de profissionais de saúde em termalismo e crenoterapia no País. Se há poucos profissionais com conhecimento na área, portanto, haverá poucas pessoas interessadas em praticar e pesquisar os benefícios e as potencialidades do termalismo em saúde. Há pouca inserção dessa temática em grades curriculares dos cursos de saúde e não há cursos regulares de especialização sobre o tema. Se antes as faculdades de medicina federais, em especial as de Minas Gerais e Rio de Janeiro, haviam cadeiras de crenologia e hidrologia médica, hoje são raríssimos os médicos termalistas no Brasil. Atualmente são disponibilizados cursos de educação à distância e/ou semipresenciais sobre práticas integrativas e complementares pelo SUS, mas até o presente momento nenhum curso voltado ao Termalismo Social e Crenoterapia foi ofertado. Sabe-se que há o interesse nessa oferta pelo Ministério da Saúde e acredita-se 
ISSN 2179-6750

que o investimento em formação básica em termalismo/crenoterapia poderia fomentar ações e serviços que se utilizem dos recursos hidrominerais naturais em benefício à saúde.

Quanto a necessidade de pesquisas sobre termalismo e crenoterapia no Brasil, estas precisam ser intensificadas. Convém ressaltar que há evidências científicas para garantir que esta área do conhecimento tem potencialidade terapêutica. Contudo, em se tratado de uma prática centenária, ou milenar, e entendendo que a ciência biomédica mudou consideravelmente nas últimas décadas, as pesquisas clínicas com práticas termais podem ser intensificadas. No panorama internacional se nota que ao longo das duas últimas décadas que o número de institutos científicos de Balneologia, especialmente nas universidades, sofreu uma diminuição considerável na Alemanha, cujo fomento direcionado para trabalhos científicos foi cortado para quase zero; em contrapartida, em alguns outros países europeus, como no caso da França, Itália, Hungria e Turquia, as pesquisas científicas relativas aos resultados clínicos de tratamentos balneológicos extensivos clássicos vêm se expandindo ${ }^{3}$. No Brasil, um edital desenvolvido pela Coordenação Geral de Áreas Técnicas do Departamento de Atenção Básica/SAS, em parceria com o Departamento de Ciência e Tecnologia/SCTIE do Ministério da Saúde e por intermédio do Conselho Nacional de Desenvolvimento Científico e Tecnológico, apoiou projetos de pesquisa sobre Práticas Integrativas e Complementares. Este edital teve como objetivo de desenvolver a pesquisa sobre PICs no Sistema Único de Saúde. Espera-se que novos editais como o referido, ou mesmo editais específicos para o termalismo/crenoterapia, sejam fomentados. Pesquisas em diversas áreas do conhecimento alusivas ao termalismo são importantes, notadamente aquelas que versam sobre os efeitos de águas minerais, pelóides e gases, especialmente no que diz respeito aos efeitos clínicos relevantes e suas quantidades necessárias para garantir efeito terapêutico ${ }^{3}$.

Outro desafio versa sobre a questão das legislações que versam sobre o termalismo/crenoterapia e águas minerais. O Código de Águas Minerais, por exemplo, é datado de 1945 e talvez pudesse ser revisitado frente aos estudos recentes, acompanhando as legislações internacionais na área. Ainda, não há legislação satisfatória sobre o funcionamento de termas em relação à saúde. O que existe está relacionada à Comissão Permanente de Crenologia do Ministério de Minas e Energia do Brasil, com pouca relação com as legislações do Ministério da Saúde, notadamente à Agência Nacional de Vigilância Sanitária (ANVISA). Convém ressaltar o exemplo português, o qual disponibiliza o Manual de Boas Práticas dos Estabelecimentos Termais. Outros países europeus destacam-se em termos legais, notadamente na França, Espanha e Alemanha, essa última com um conjunto de padrões de qualidade conhecido como Begrisbestimmungen und Qualitätsstandards für die Prädikatisierung von Kurorten, Erholungsorten und Heilbrunnen 317 
ISSN 2179-6750

(Definições e padrões de qualidade para spas, resorts e fontes minerais), que leva em conta a concentração de minerais nas fontes e os métodos de análise e controle de qualidade de infraestrutura de estâncias de tratamento termal ${ }^{3}$.

O fortalecimento dos estabelecimentos de saúde com termalismo e crenoterapia são necessários. O Brasil possui grandes complexos turísticos com águas termais, em sua grande maioria voltados apenas ao lazer e pouca inserção em saúde. Boa parte dos tratamentos desenvolvidos em estâncias termais convergem para o bem-estar e estética, com pouca relação à recuperação e manutenção da saúde. Embora há técnicas crenoterápicas podem ser realizadas foram das termas, o termalismo/balneoterapia clássico se realizam em termas/balneários. Há balneários tradicionais que se encontram em situação de abandono. Revitalizar os balneários termais, especialmente os públicos e históricos, estão entre alguns desafios para revigorar o termalismo em saúde no Brasil, bem como resgatar a memória e a história do termalismo brasileiro.

Para tanto, faz-se necessário identificar, cadastrar e fortalecer os estabelecimentos de saúde com termalismo e crenoterapia. Apenas doze estabelecimentos de saúde que oferecem práticas de termalismo e crenoterapia estão cadastrados, até início de 2014, no Cadastro Nacional de Estabelecimentos de Saúde. Destes, a grande parte são privados. Contudo, sabe-se que da existência de outros serviços ainda não cadastrados, por isso faz-se necessário acompanha-los em termos de produção de práticas terapêuticas. Também são poucos os locais com experiências em Termalismo Social no Sistema Único de Saúde, sobretudo, como dito, por contar com poucos profissionais qualificados para o assunto. Apesar disso, após a Política Nacional de Práticas Integrativas e Complementares esta realidade tende a mudar, porém não será de forma satisfatória se o financiamento específico para as práticas termais permanecerem inexistente.

O termo "termalismo social" denota o acesso democrático às termas para tratamento, especialmente após a década de 1950. Na França, o ano de 1947 marca o início do reembolso dos tratamentos termais pela Seguridade Social e o crescimento do termalismo social medicalizado no país ${ }^{16}$. Os curistas tem assegurado o benefício social do reembolso dos cuidados termais e dos honorários do médico termal que desenvolve os cuidados. Quando a renda anual do paciente é inferior a $€ 14.000$ (dados de 2014), o mesmo pode também receber um reembolso parcial de transporte, com base no preço do bilhete da passagem, bem como do alojamento na estância, sendo o tempo do tratamento (cura termal) obrigatoriamente três semanas (18 dias de tratamento) ${ }^{16}$. O reembolso dos tratamentos termais ainda hoje é praticado na França, embora existam discussões as quais apontam problemas no sentido de fraudes nas indicações médicas para usuários do sistema de tratamentos. Na Alemanha a maior parte das intervenções em estancias termais não são mais 
ISSN 2179-6750

cobertas pelos planos de saúde, tendo em conta a longa permanência nas estâncias e, portanto, dos altos custos alinhados à falta de liquidez geral nos sistemas de saúde ${ }^{3}$. Em Portugal o copagamento aos tratamentos termais foi cortado em 2011 por conta da crise financeira vivenciada. Contudo, no X Congresso da Sociedade Portuguesa de Hidrologia Médica, ocorrida entre os dias 19 e 20 de maio de 2017 em Caldas da Rainha, o Ministro da Saúde esteve presente e assinalou que o retorno do copagamento está sendo estudado. Os pagamentos/copagamentos dos tratamentos termais colaboram para a manutenção das termas. No Brasil ainda se está por estudar uma forma de financiamento que seja economicamente viável para o SUS, o qual já se encontra em um crônico subfinanciamento, agravado após a Emenda Constitucional 95 de 2016.

No caso Brasileiro, por serem as águas termais recursos subterrâneos, o Estado é detentor de tais águas e, portanto, deveria o mesmo garantir meios sustentáveis do uso desse recurso natural para balneários públicos, ou como cotas dos balneários privados, de modo a garantir os tratamentos termais. Boa parte das cidades termais possuem apenas modelos privados de exploração da água, com escasso acesso público. Soma-se ao fato a vulnerabilidade de certas estâncias hidrominerais brasileiras frente à fraca legislação de proteção ambiental das áreas e da expansão do mercado de águas minerais engarrafadas ${ }^{17}$.

\section{Conclusão}

Após a Política Nacional de Práticas Integrativas e Complementares o termalismo social/ crenoterapia no cuidado à saúde tem se fortalecido, ainda que de maneira tímida, revelando seu potencial terapêutico de recuperar, manter e promover a saúde. Para além dos aspectos biológicos que as propriedades terapêuticas das águas minerais naturais proporcionam em balneoterapia, o Termalismo Social pode potencializar as cidades termais históricas, também novas, no sentido de emprego e renda, proteção dos recursos naturais e cultura de saúde.

Destaca-se que o Termalismo/Balneoterpaia/Crenoterapia engloba ainda outros recursos terapêuticos como os pelóides, lamas, e no SUS podem se configurar em duas vertentes: uma hidrologia terapêutica que contemple práticas as quais podem ser utilizadas nas Unidades Básicas de Saúde, não apenas em termas e balneários específicos, como também o Termalismo clássico, enquadrado na figura do curistas que vai às termas para um período específico de cuidados integrais à saúde. Para ambas as vertentes, um conjunto de ações é necessário para fomentar o Termalismo Social/crenoterapia, notadamente a informação e a formação do/para profissionais de saúde sobre a temática, investimento em pesquisas, elaboração de diretrizes e financiamento para a manutenção dos balneários e aplicação das práticas termais e crenoterápicas em diferentes níveis de atenção. 


\section{Referências}

1. Ministério da Saúde (BR), Secretaria de Atenção à Saúde, Departamento de Atenção Básica. Política Nacional de Práticas Integrativas e Complementares no SUS - PNPIC-SUS. Brasília, DF: Ministério da Saúde, 2006.

2. Hellmann F. Termalismo social no Sistema Único de Saúde: ampliando ações e olhares quanto ao uso terapêutico da água. Cad Naturol Terap Complem. 2014;3(5):9-11. http://dx.doi.org/10.19177/cntc.v3e520149-11.

3. Gutenbrunner C. Balneologia e estâncias hidrominerais na Alemanha. In: Hellmann F, Rodrigues DMO. Termalismo e Crenoterapia no Brasil e no Mundo. Palhoça, SC: Editora UNISUL; 2017. p. 187-97.

4. Gutenbrunner C; Bender T, Cantista P, Karagülle Z. A proposal for a worldwide definition of health resort medicine, Balneology, medical hydrology and climatology. Int J Biometeorol. 2010 Sep;54(5):459-507. http://dx.doi.org/10.1007/s00484-010-0321-5.

5. Lemaire J. Social thermalism in Belgium: "la prévoyance sociale" and its instituicions: the fruit of cooperation. Annals of Public and Cooperative Economics, 1956 Jan;27(1):1-13. https://doi.org/10.1111/j.1467-8292.1956.tb01226.x.

6. Silva MA. O Complexo Termal da Serra de Caldas: a linguagem do contemplativo e do imaginário sobre espaços de Goiás. Geoambiente Online. 2009;(13):1-24. https://doi.org/10.5216/rev.\%20geoambie.v0i13.25997.

7. Quintela MM. Cura termal: entre as práticas “populares" e os saberes “científicos". Trabalho apresentado no: VIII Congresso Luso-Afro-Brasileiro de Ciências Sociais; 2004 setembro 16-18 [acesso em 2017 abr 2017]; Coimbra, Portugal. Coimbra: Universidade de Coimbra; 2004.

http://www.ces.uc.pt/lab2004/inscricao/pdfs/painel37/MariaManuelQuintela.pdf

8. Untura Filho M. La historia del termalismo en Brasil. Na Hidrol Med. 2010;3:35-44.

9. Martins AM, Mansur, KL, Pimenta TS, Caetano LC. Crenoterapia das Águas Minerais do Estado do Rio de Janeiro. Rio de Janeiro: Departamento de Recursos Minerais, 2006 [acesso em 2017 abr 2] Disponívek em http://www.drm.rj.gov.br/index.php/areas-de-atuacao/28aguaminara/56-aguamineral

10. Franco AM. Os primeiros registros do uso de águas termais e a formação das estâncias hidrominerais no Brasil. Cad Naturol Terap Complem. 2014:3(5):9-11. http://dx.doi.org/10.19177/cntc.v3e5201429-40. 
ISSN 2179-6750

11. Marrichi JMO. A cidade termal: ciência das águas e sociabilidade moderna entre 1839 a 1931. São Paulo: Annalube; 2015. 221 p.

12. Dahdal AB. Termas de Águas de Lindóia: uma visão contemporânea dos usuários sobre o balneário nos aspectos termal e turístico [dissertação]. Campinas: Universidade Estadual de Campinas; 2014.

13. Ministério da Saúde (BR). Política nacional de práticas integrativas e complementares no SUS: atitude de ampliação de acesso. 2a. ed. Brasília, DF: Ministério da Saúde; 2015. 96 p.

14. Organización Mundial de la Salud. Medicina tradicional: $111^{\circ}$ reunión, 24 de enero de 2003 [acesso 2017 abr 2]. [local desconhecido]: WHO. Disponível em: http://apps.who.int/iris/bitstream/10665/79679/1/seb111r12.pdf?ua=1

15. Hellmann F, Denes K, Termalismo e Crenoterapia no Brasil. In: Hellmann F, Rodrigues DMO. Termalismo e crenoterapia no Brasil e no mundo. Palhoça, SC: Editora UNISUL; 2017. p. 166-186.

16. Forestie R, Tabone W, Palmer M, Jeambrun P, Chareyras J, Guerrer D, et al. A Crenobalneoterapia na França: visão geral. In: Hellmann F, Rodrigues DMO. Termalismo e Crenoterapia no Brasil e no Mundo. Palhoça, SC: Editora UNISUL; 2017. p. 166-86.

17. Ninis AB, Drummond JA. Áreas (des)protegidas do Brasil: as estâncias hidrominerais. Ambient Soc. 2008:11(1):149-66. http://dx.doi.org/10.1590/S1414-753X2008000100011. 